\title{
Cisplatin-induced expression of Gb3 enables verotoxin- I treatment of cisplatin resistance in malignant pleural mesothelioma
}

cells

\author{
D Johansson', C Andersson', J Moharer', A Johansson ${ }^{2}$ and P Behnam-Motlagh", ${ }^{*, 3}$ \\ 'Department of Medical Biosciences, Clinical Chemistry, Umeå University, Umeå S-901 85, Sweden; ${ }^{2}$ Department of Odontology, Periodontology, Umeå \\ University, Umeå S-901 85, Sweden; ${ }^{3}$ Department of Radiation Sciences, Oncology, Umeå University, Umeå S-901 85, Sweden
}

BACKGROUND: A major problem with cisplatin treatment is the development of acquired-drug resistance of the tumour cells. Verotoxin-I (VT-I) exerts its cytotoxicity by targeting the membrane glycolipid globotriasosylceramide (Gb3), a molecule associated with drug resistance. Cisplatin- and VT-I-induced apoptosis involves mitogen-activated protein kinase (MAPK) activation, and deactivation of MAPKs is associated with cisplatin resistance. This study aimed to investigate whether a sub-toxic concentration of VT-I could enhance cisplatin-induced apoptosis and overcome acquired-cisplatin resistance in cultured cancer cell lines.

METHOD: P3I and HI299 cells with corresponding cisplatin-resistant sub-lines (P3I res/HI299res) were incubated with VT-I and/or cisplatin followed by determination of Gb3 expression, cell viability, apoptosis, and signalling pathways.

RESULTS: Cells from the resistant sub-lines had elevated Gb3 expression compared with the parental cell lines, and cisplatin further increased Gb3 expression, whereas VT-I reduced the percentage of Gb3-expressing cells. Combination of cisplatin and sub-toxic concentrations of VT-I led to a super-additive increase of cytotoxicity and TUNEL staining, especially in the cisplatin-resistant sublines. Blockade of Gb3 synthesis by a Gb3 synthesis inhibitor not only led to eradicated TUNEL staining of P3I cells, but also sensitised P3I res cells to the induction of apoptosis by cisplatin alone. Cisplatin- and VT-I-induced apoptosis involved the MAPK pathways with increased C-Jun N-terminal kinase and MAPK kinase-3 and -6 phosphorylation.

CONCLUSIONS: We show the presence of Gb3 in acquired-cisplatin resistance in P3I res and HI299res cells. Cisplatin up-regulated Gb3 expression in all cells and thus sensitised the cells to VT-I-induced cytotoxicity. A strong super-additive effect of combined cisplatin and a sub-toxic concentration of VT-I in cisplatin-resistant malignant pleural mesothelioma cells were observed, indicating a new potential clinical-treatment approach.

British Journal of Cancer (2010) I 02, 383-39I. doi:I0.I038/sj.bjc.6605467 www.bjcancer.com

Published online 15 December 2009

(c) 2010 Cancer Research UK

Keywords: acquired resistance; apoptosis; cisplatin; Gb3; mesothelioma; verotoxin- I

Lung cancer is the first and second leading cause of cancer-related death in men and women, respectively (Parkin, 2001; Jemal et al, 2003). The most common type is non-small cell lung cancer (NSCLC), which accounts for over $75 \%$ of all cases (Brognard et al, 2001). Asbestos can cause a variety of lung diseases including lung cancer and pleural mesothelioma. Malignant pleural mesothelioma (MPM) is a highly mortal malignancy with poor prognosis partially because of treatment resistance (Leard and Broaddus, 2004). Treatment options are surgery, radiotherapy, and chemotherapy often including platinum-based drugs such as cisplatin (cisdiamminedichloroplatinum (II),), which is an extensively used anticancer drug. Cisplatin acts at least in part, by formation of platinum-DNA adducts, which hinders rapidly dividing cells from

*Correspondence: Dr P Behnam-Motlagh, Department of Medical Biosciences, Clinical Chemistry, Building 6M, 2nd floor, Umeå S-90I 85, Sweden; E-mail: parviz.behnam@medbio.umu.se

Received 22 June 2009; revised 26 October 2009; accepted 9 November 2009; published online 15 December 2009 duplicating their DNA for mitosis and activation of apoptosis (Zwelling et al, 1979; Siddik, 2003). However, a major problem with cisplatin treatment is the development of acquired-drug resistance of the cancer cells (Andrews and Howell, 1990; Kasibhatla and Tseng, 2003) involving increased MDR1/PgP activity (Zhou, 2008). Some mechanisms of cisplatin resistance include reduction of platinum accumulation by alteration of transmembrane pumps, enhancement of DNA damage repair, and reduced apoptosis induction (Krishan et al, 1997; Cvijic et al, 1998; Ohmichi et al, 2005; Liu et al, 2007). Mitogen-activated protein kinases (MAPKs) are activated in cisplatin-induced apoptosis in most investigated cell systems and induced cisplatin resistance is also associated with reduced activation of MAPKs (Brozovic and Osmak, 2007).

Globotriasosylceramide (Gb3) consist of a trisaccharide linked to a lipid based in the plasma membrane and is expressed by several tumour cell lines originating from breast cancer, ovarian cancer, colon carcinoma, haematological malignancies, and astrocytoma tumours as well as normal endothelial and epithelial cells (LaCasse et al, 1999; Gariepy, 2001; Kovbasnjuk et al, 2005; 
Johansson et al, 2006). MDR1/PgP acts as a glycolipid translocase involved in the biosynthesis of glycolipids such as Gb3, and elevated levels of Gb3 have also been seen in drug-resistant cancers, and functional interplay between membrane $\mathrm{Gb} 3$ and MDR1/PgP has been suggested (Lingwood et al, 1998; De Rosa et al, 2008). Gb3 functions as cell surface receptor for verotoxin-1 (VT-1, Shiga-like toxin-1) produced by pathogenic strains of Escherichia coli (Lingwood et al, 1987; Jacewicz et al, 1989; Rose and Clark, 1989).

VT-1 has one enzymatically active part (A), and one part that binds to the cell surface (B). The B part consists of five identical sub-units, which can all bind to the $\mathrm{Gb} 3$ receptor in which the $\mathrm{A}$ sub-unit is internalised and cytotoxic through ribosome inactivation (Endo et al, 1988; Olsnes and Sandvig, 1988; Saxena et al, 1989; O'Brien et al, 1992; Gariepy, 2001; Sandvig et al, 2002). VT-1 has shown efficacy against meningioma, astrocytoma, as well as renal tumour xenografts in mice (Arab et al, 1999; Salhia et al, 2002; Ishitoya et al, 2004). The B part of VT-1 has also been suggested as a novel approach to deliver other anti-tumour agents (Vingert et al, 2006).

A sub-toxic concentration of VT-1 could possibly enhance cisplatin-induced apoptosis and overcome acquired-cisplatin resistance in cultured cancer cell lines, as MAPKs are involved in apoptosis induction of both agents and in cisplatin resistance. The aim of the study was to quantify Gb3 expression in cisplatinsensitive and -resistant MPM and NSCLC cell lines, and to investigate the potential of using VT-1 or agent adhered to its $\mathrm{B}$ sub-unit as highly potent and specific agents to overcome acquired-cisplatin resistance.

\section{MATERIALS AND METHODS}

\section{Cell lines and cell culture}

Two human cancer cell lines were used: P31 (Marklund et al, 1982), an MPM and H1299 (American Type Culture Collection, CRL-5803), an NSCLC cell line as well as corresponding sub-lines with acquired-cisplatin resistance (P31res and H1299res). The cells were maintained under standard cell culture conditions, grown as monolayer culture in Eagle's MEM in Earl's salt (Gibco Ltd, Paisley, Scotland, UK) supplemented by $10 \%$ foetal bovine serum (Biochrom KG, Berlin, Germany) and $200 \mu \mathrm{moll}^{-1}$ L-glutamine. They were incubated at $37^{\circ} \mathrm{C}$ in a humidified atmosphere containing $5 \% \quad \mathrm{CO}_{2}$. Medium of the resistant sub-lines was between experiments supplemented by either $1.2 \mathrm{mgl}^{-1}$ (P31res) or $2 \mathrm{mgl}^{-1}$ (H1299res) cisplatin.

\section{Determination and inhibition of Gb3 and MDR1/PgP expression of cultured cells}

The cellular expression of $\mathrm{Gb} 3$ of the cell lines was identified by a monoclonal rat IgM antibody (Immunotech, Marseille, France) and MDR1/PgP by a monoclonal antibody from Chemicon Internat. Inc. (Temecula, CA, USA) on an FACS Calibur flow cytometer (Becton Dickinson Immunocytometry Systems, San Jose, CA). DL-threo-1-phenyl-2-palmitoylamino-3-morpholino-1propanol, PPMP (Sigma-Aldrich, St Louis, MO, USA), a chemical inhibitor of glucosylceramide synthesis, was used to deplete Gb3 expression by culturing cells with $2 \mu \mathrm{moll}^{-1}$ PPMP for $72 \mathrm{~h}$. Verapamil was from Abbott Laboratories, Abbott Part, IL USA, and cyclosporin A from Sigma-Aldrich.

\section{Cell viability assay}

A fluorometric method using fluorescein diacetate (Amersham International, Amersham, UK) was used to quantify cell viability and determine VT-1 (Sigma-Aldrich) sensitivity of P31 and H1299 cells in vitro. Cells $\left(1 \times 10^{4}\right)$ were plated with $100 \mathrm{mgl}^{-1}$ medium in the wells of 96-well microtiter plates. The plates were first incubated at $37^{\circ} \mathrm{C}$ for $24 \mathrm{~h}$ with culture medium only, then medium was replaced with fresh medium, containing (1) $0.1-5.0 \mu \mathrm{gl}^{-1}$ (P31) or $0.001-1.0 \mu \mathrm{gl}^{-1}$ (H1299) VT-1 or (2) $0.1-10.0 \mathrm{mgl}^{-1}$ cisplatin with or without VT-1 $\left(0.1 \mu \mathrm{gl}^{-1}\right.$ for P31, $0.001 \mu \mathrm{gl}^{-1}$ for H1299). The incubation was continued for $72 \mathrm{~h}$, and then the medium was removed by flicking the plate, and wells were washed once with $200 \mu$ l PBS buffer. To each well was then added $150 \mu \mathrm{l}$ of PBS containing $10 \mathrm{mgl}^{-1}$ fluorescein diacetate, and the plates incubated for $45 \mathrm{~min}$ at $37^{\circ} \mathrm{C}$, followed by fluorescence determination in a fluorometer (LS 55, Perkin Elmer, MA, USA) using 485 and $538 \mathrm{~nm}$ for excitation and emission, respectively.

\section{Flow cytometry analysis}

P31res and H1299res cells were trypsinised and suspended in PBS and double stained with Gb3 goat anti-rat IgM (Immunotech) and MDR1/PgP IgG $\mathrm{Ia}_{2 \mathrm{a}}$ anti-mouse primary antibodies (Chemicon Millipore, MA USA). Controls were derived by incubating the cells with corresponding rat $\operatorname{IgM}$ and mouse $\operatorname{IgG}_{2 a}$ isotype antibodies (Invitrogen, Carlsbad, CA, USA) for $1 \mathrm{~h}$ at $4^{\circ} \mathrm{C}$, followed by washing and centrifuging for $10 \mathrm{~min}$ with PBS-BSA. Subsequently, cell pellets were re-suspended and incubated with secondary antibody goat anti-rat IgM and goat anti-mouse $\mathrm{IgG}_{2 \mathrm{a}}$ (Invitrogen) for $1 \mathrm{~h}$ at $4^{\circ} \mathrm{C}$. After washing the cells and centrifuging for $10 \mathrm{~min}$, cells were analysed with an FACScan flow cytometer (Becton Dickinson, San Jose, CA, USA) on channels FL4 or FL1 and data processed using BD Cell Quest software. After gating out debris and cell clumps, the data were plotted as area histograms.

Terminal deoxynucleotidyl transferase (TdT)-mediated dUTP nick end labelling (TUNEL) staining detecting apoptosis-specific nuclear DNA fragmentation was used as a marker for late stage apoptosis. Free $3^{\prime}-\mathrm{OH}$ terminal was labelled with modified fluorescence-labelled nucleotides (dUTP) by catalysis of TdT. Roche's in situ cell death detection kit, TMR red (Roche, Mannheim, Germany), was used. P31 and H1299 cells were cultured to about $80 \%$ confluence and the medium was thereafter changed to fresh medium containing 0 or $5 \mathrm{mgl}^{-1}$ cisplatin and/or $0.1 \mu \mathrm{gl} \mathrm{l}^{-1} \mathrm{VT}-1$, and incubation continued for $72 \mathrm{~h}$. Cells were thereafter harvested with trypsin and any floating cells were collected by centrifugation. Cells were then TUNEL stained according to the manufactures instructions and TUNEL staining was determined by flow cytometry.

\section{Caspase activity determination}

Fluorometric activity assays measuring caspase-3, -8, and -9 enzyme activities were used (R\&D Systems Inc. MN, USA). P31 and $\mathrm{H} 1299$ cells were treated with 0 or $5 \mathrm{mgl}^{-1}$ cisplatin and/or $0.1 \mu \mathrm{g}^{-1} \mathrm{VT}-1$ for $24 \mathrm{~h}$ and thereafter lysed in lysis buffer for $10 \mathrm{~min}$. Cell lysates (total protein concentration 100-200 $\mu \mathrm{g}$ ) were incubated with caspase- $3,-8$, or -9 fluorogenic caspase-specific substrate at $37^{\circ} \mathrm{C}$ for $2 \mathrm{~h}$. The fluorescence signal was determined with an LS55, Luminescence spectrometer (Perkin Elmer) using 400 and $505 \mathrm{~nm}$ excitation and emission wavelengths, respectively. Total protein content was determined with bicinchoninic acid Protein Assay kit (Pierce Biotechnology Inc., IL, USA).

\section{SDS-PAGE gel electrophoresis and immunoblotting}

VT-1 influence on specific proteins involved in apoptosis signal transduction was investigated through western blotting. Cells were exposed to 0 or $5 \mathrm{mgl}^{-1}$ cisplatin and/or $0.1 \mu \mathrm{gl}^{-1} \mathrm{VT}-1$ for $24 \mathrm{~h}$ and then lysed in lysis buffer (R\&D Systems Inc.). Cell extract was incubated with NuPAGE $4 \times$ LDS sample buffer and NuPAGE 
reducing agent for $10 \mathrm{~min}$ at $100^{\circ} \mathrm{C}$. Samples $\left(19.5 \mu \mathrm{gl}^{-1}\right.$ protein) were run on a $12.5 \%$ Tris - $\mathrm{HCl}$ SDS-PAGE criterion precast gel (Bio-Rad, Hercules, CA, USA) using $1 \times$ MOPS buffer and NuPage antioxidant. The buffers, reducing agent, and antioxidant were from Invitrogen. Blotting was performed onto Immune-Blot PVDF membranes (Bio-Rad). Membrane was then blocked in TBS buffer containing $0.2 \%$ Tween $20,20 \mathrm{mmoll}^{-1}$ Tris $\mathrm{pH} \quad 7.4$, and $150 \mathrm{mmoll}^{-1} \mathrm{NaCl}$ milk RT for $1 \mathrm{~h}$. Thereafter, the membrane was incubated overnight with primary antibody against Akt, p-Akt (ser473 and tyr308), Bad, p-Bad (ser 136 and 112) Bid, Bim, C-Jun N-terminal kinase (JNK) (1 and 2), p-JNK (1 and 2), MCL-1, MAPK kinase-3 (MKK-3), p-MAPK kinase-3 and -6 (MKK3/6), P44/42, p-P44/42, or PUMA and after repeated washing with TBS buffer, the secondary antibodies both diluted in 5\% milk in TBS buffer and $0.25 \%$ Tween 20 were incubated for $1 \mathrm{~h}$. Membrane was then washed again with TBS buffer, and antibody detection was performed by enhanced chemiluminescence staining (ECL Advance western blotting detection system, Amersham Biosciences, Buckinghamshire, UK). Monoclonal $\beta$-actin antibody was used for detection of actin as loading control. All antibodies were from Cell Signalling Technology Inc. (Danvers, MA, USA).

\section{Statistics}

Statistical significance was tested with one-way ANOVA. The level of significance for rejecting the null hypothesis of zero-treatment effect was taken to be $P=0.05$.

\section{RESULTS}

\section{Basal expression of Gb3 and the effect of cisplatin on MPM and NSCLC cells}

Cell surface expression of Gb3 was evaluated by FACS analysis using monoclonal anti-Gb3 antibodies. Low levels of Gb3expressing cells was found in P31 (1\%) and H1299 (12\%) cells, but expression was elevated in the cisplatin-resistant sub-lines to $27 \%$ and $29 \%$, respectively (Figure 1 ). Incubation of cells with
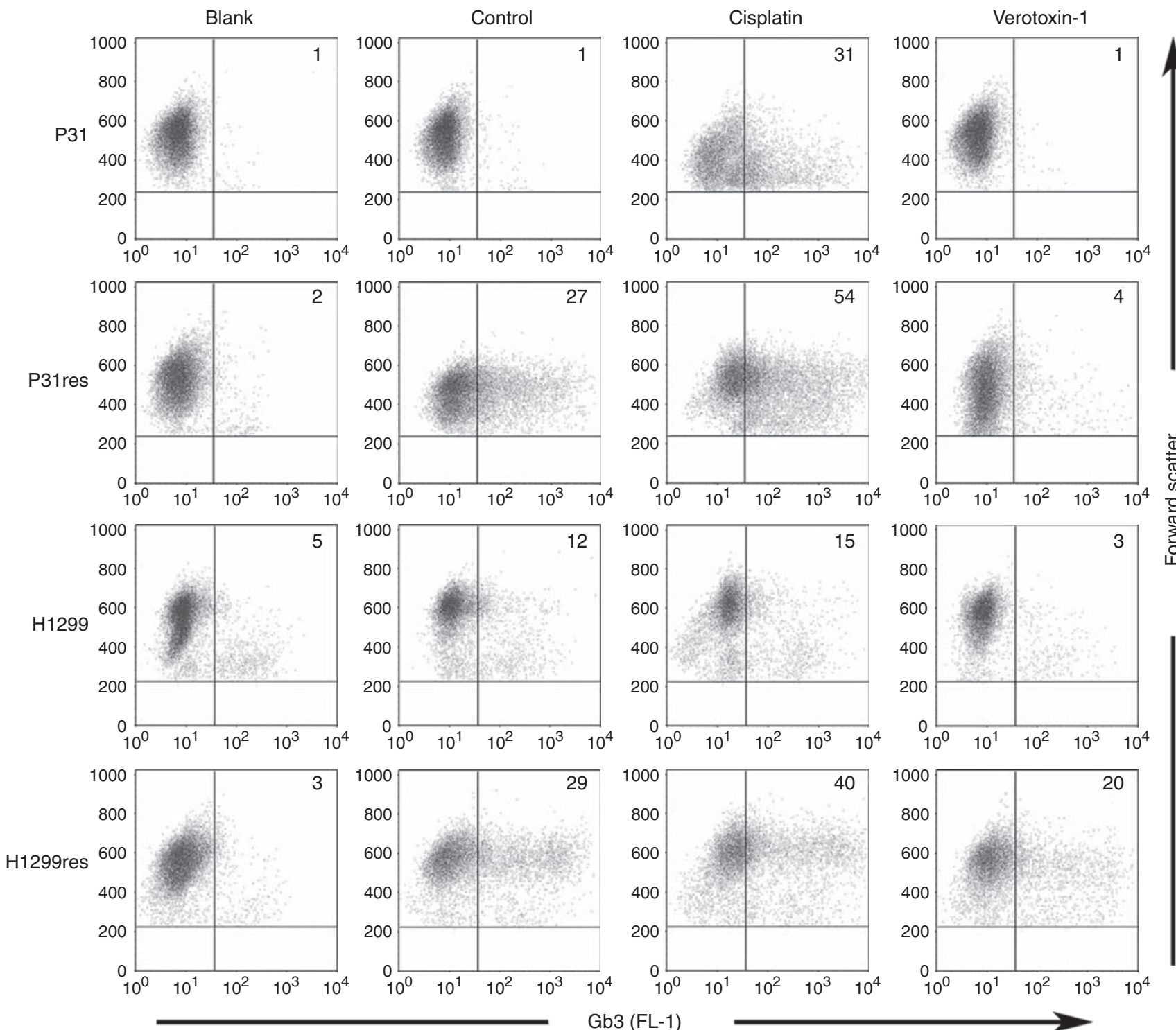

Figure I Flow cytometry analysis of Gb3 expression in P3I and HI299 cells. Gb3 expression in cells not incubated with and cells incubated for $72 \mathrm{~h}$ with $5 \mathrm{mgl}^{-1}$ cisplatin or $\left.0.1 \mu \mathrm{gl}\right|^{-1} \mathrm{VT}$-I, respectively. The percentage of Gb3-expressing cells is noted in the right quadrant in each dot plot. Blank shows unspecific secondary anti-body binding, whereas control shows cell not incubated with either cisplatin or VT-I. Representative results out of at least three independent experiments are shown. 

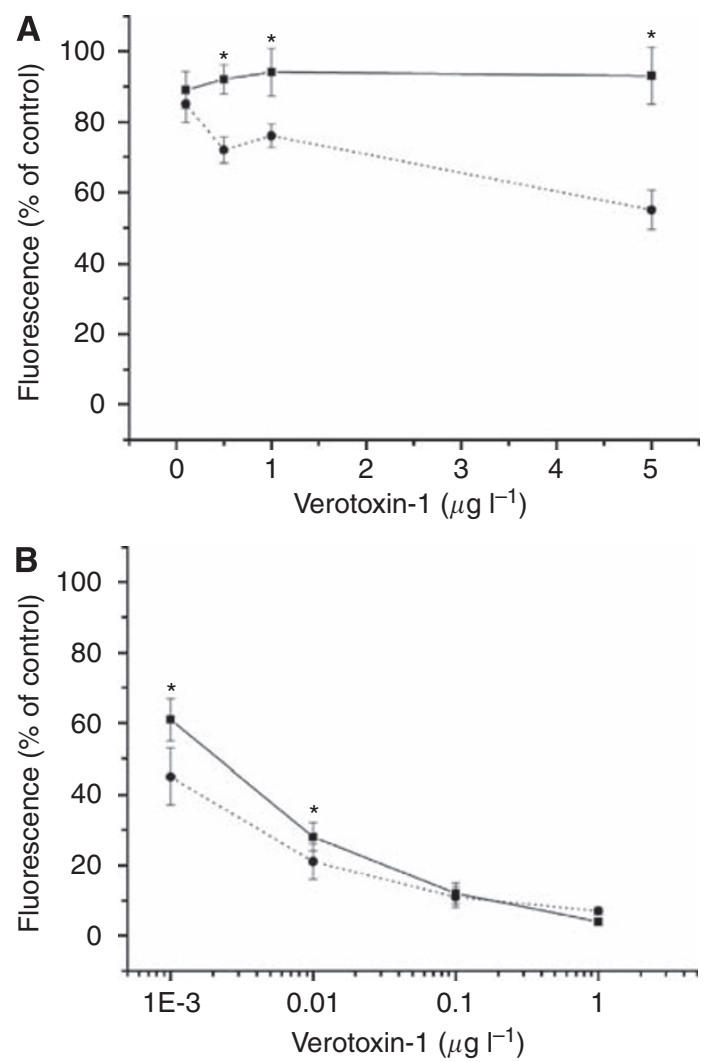

Figure 2 Cell viability (FMCA assay) after exposure to increasing concentrations of VT-I for $72 \mathrm{~h}$. (A) P3| (straight line) and P3I res (dotted line) cells exposed to $0.1-5.0 \mu \mathrm{gI} \mathrm{I}^{-1} \mathrm{VT}-\mathrm{I}$. (B) HI299 (straight line) and HI299res (dotted line) cells exposed to $\left.0.00|-| \mu g\right|^{-1} V T-I$. Significant differences $(P<0.05)$ between cell lines is indicated $(*)$. Mean \pm s.d. $(n=3)$.
$5 \mathrm{mgl}^{-1}$ cisplatin for $72 \mathrm{~h}$ increased Gb3 expression to $31 \%$ and $54 \%$ in P31 and P31res cells, and to $15 \%$ and $40 \%$ in $\mathrm{H} 1299$ and H1299res, respectively. Incubation with $0.1 \mu \mathrm{g} 1^{-1} \mathrm{VT}-1$ reduced the percentage of Gb3-expressing P31 and P31res cells to $1 \%$ and $4 \%$, respectively, and incubation with $0.001 \mu \mathrm{g} \mathrm{l^{-1 }} \mathrm{VT}-1$ markedly reduced the percentage of Gb3-expressing H1299 and H1299res cells to $3 \%$ and $20 \%$, respectively (Figure 1 ).

\section{VT-1 and cisplatin cytotoxicity on MPM and NSCLC cells}

Exposure of the MPM cells to $0.1-5 \mu \mathrm{gl} 1^{-1} \mathrm{VT}-1$ for $72 \mathrm{~h}$ showed no cytotoxicity of the toxin to P31 cells and a modest cytotoxicity on P31res cells, whereas both NSCLC cell sub-types were sensitive to VT-1 in concentrations as low as $1 \times 10^{-3} \mu \mathrm{gl}^{-1}$ (Figure 2). Incubation with $0.1-10 \mathrm{mgl}^{-1}$ cisplatin for $72 \mathrm{~h}$ reduced cell viability in P31 and H1299 cells concentration dependently and as expected less so in the cisplatin-resistant sub-lines (Figure 3). Combination of cisplatin and VT-1 $\left(0.1\right.$ or $\left.0.001 \mu \mathrm{gl}^{-1}\right)$ led to a significant increase in cytotoxicity, especially in the cisplatinresistant sub-lines (Figure 3).

\section{MDR1/PgP and Gb3 expression of cells and their resistant cell sub-lines}

Flow cytometry showed a correlation between MDR1/PgP and Gb3 co-expression in P31res as well as H1299res cell sub-lines (Figure 4). P31 res cells showed co-expression in two sub-fractions with one expressing $\sim 10$-fold expression of MDR1/PgP compared with Gb3. Incubation of the cells with $10 \mu \mathrm{moll}^{-1}$ verapamil for $72 \mathrm{~h}$ before expression analysis did, however, not reduce the expression of MDR1/PgP or Gb3 (results not show).

We, therefore, also tested whether the more effective MDR1/PgP inhibitor cyclosporin A $\left(10 \mu \mathrm{moll}^{-1}\right.$ incubated with the cells for $72 \mathrm{~h})$ as well as PPMP $\left(2 \mu \mathrm{moll}^{-1}\right)$ affected the co-expression of MDR1/PgP and Gb3. Un-expectantly, cyclosporin A did not
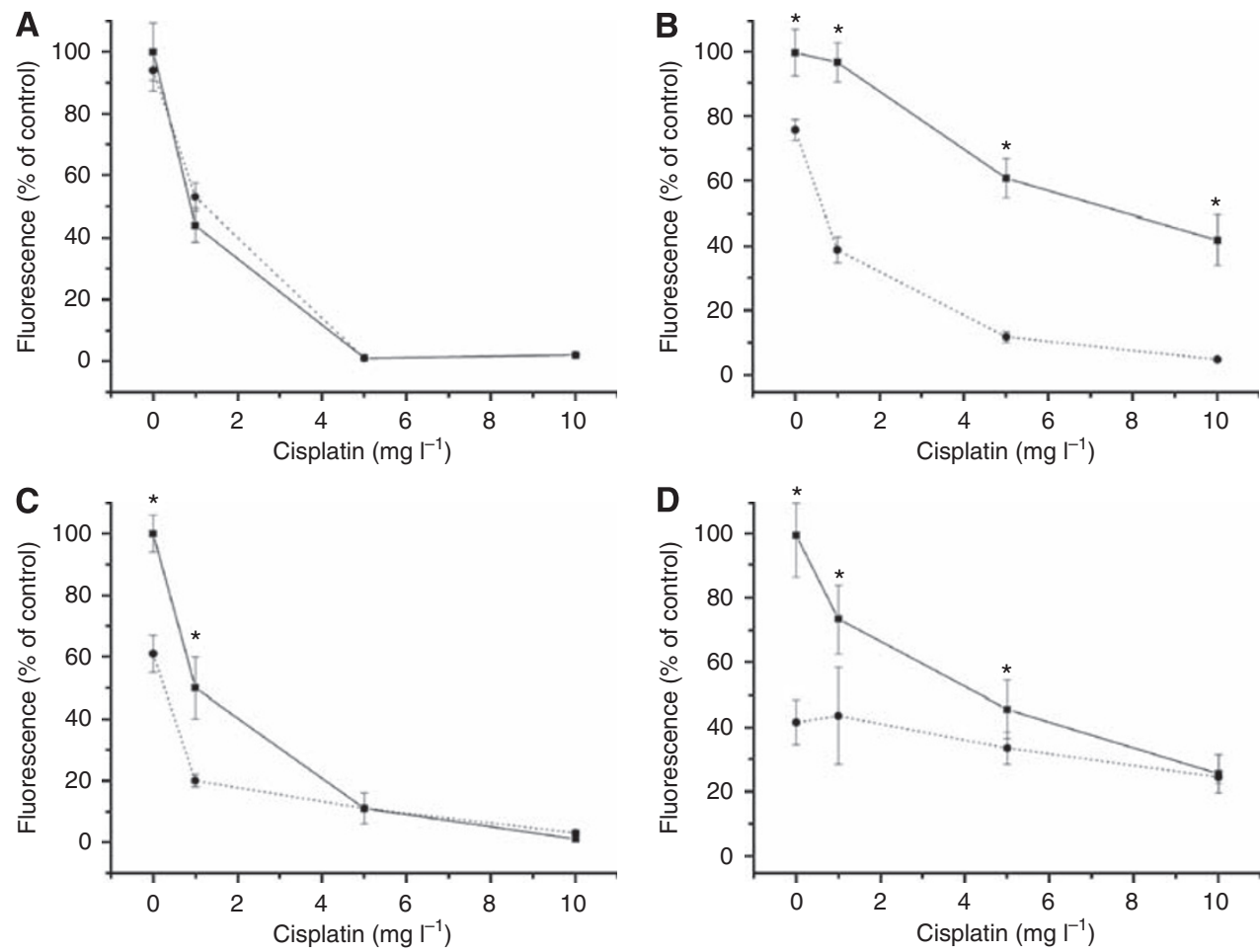

Figure 3 Cell viability (FMCA assay) after exposure of MPM and NSCLC cells to $0.1-\left.10 \mathrm{mg}\right|^{-1}$ cisplatin alone (filled line) or in combination with $0.1 \mu \mathrm{g}^{-1}$ (P3I sub-lines) or $\left.0.001 \mu \mathrm{g}\right|^{-1}$ (HI299 sub-lines) of VT-I (dotted line) for $72 \mathrm{~h}$. (A) P3Icells, (B) P3Ires cells, (C) HI299t cells, and (D) HI299res cells. Significant differences $(P<0.05)$ between cell lines with cisplatin alone and when combined with VT-I is indicated $(*)$. Mean \pm s.d. $(n=3)$. 

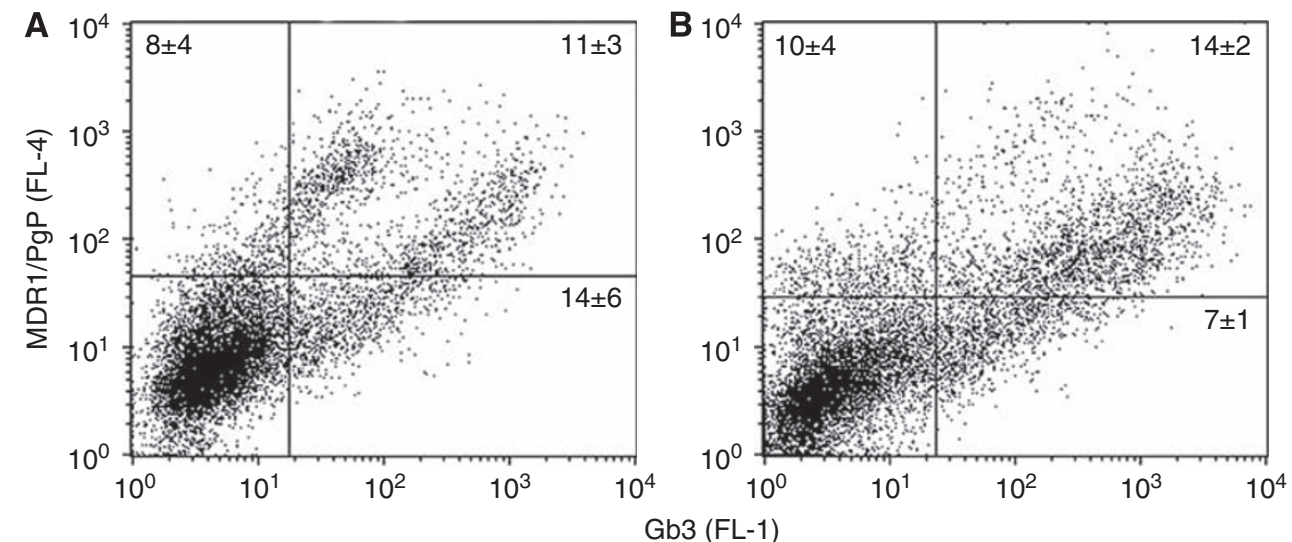

Figure 4 Flow cytometry analysis of Gb3- and MDRI/Pgp expression of cisplatin-resistant cell sub-lines. (A) Cell surface expression of MDRI/PgP (ordinate) and Gb3 (abscissa) of P3Ires cells. (B) Cell surface expression of MDRI/PgP (ordinate) and Gb3 (abscissa) of HI299res cells. Representative results are shown and the percentage mean $(n=3)$ of stained cells is noted in each dot plot.
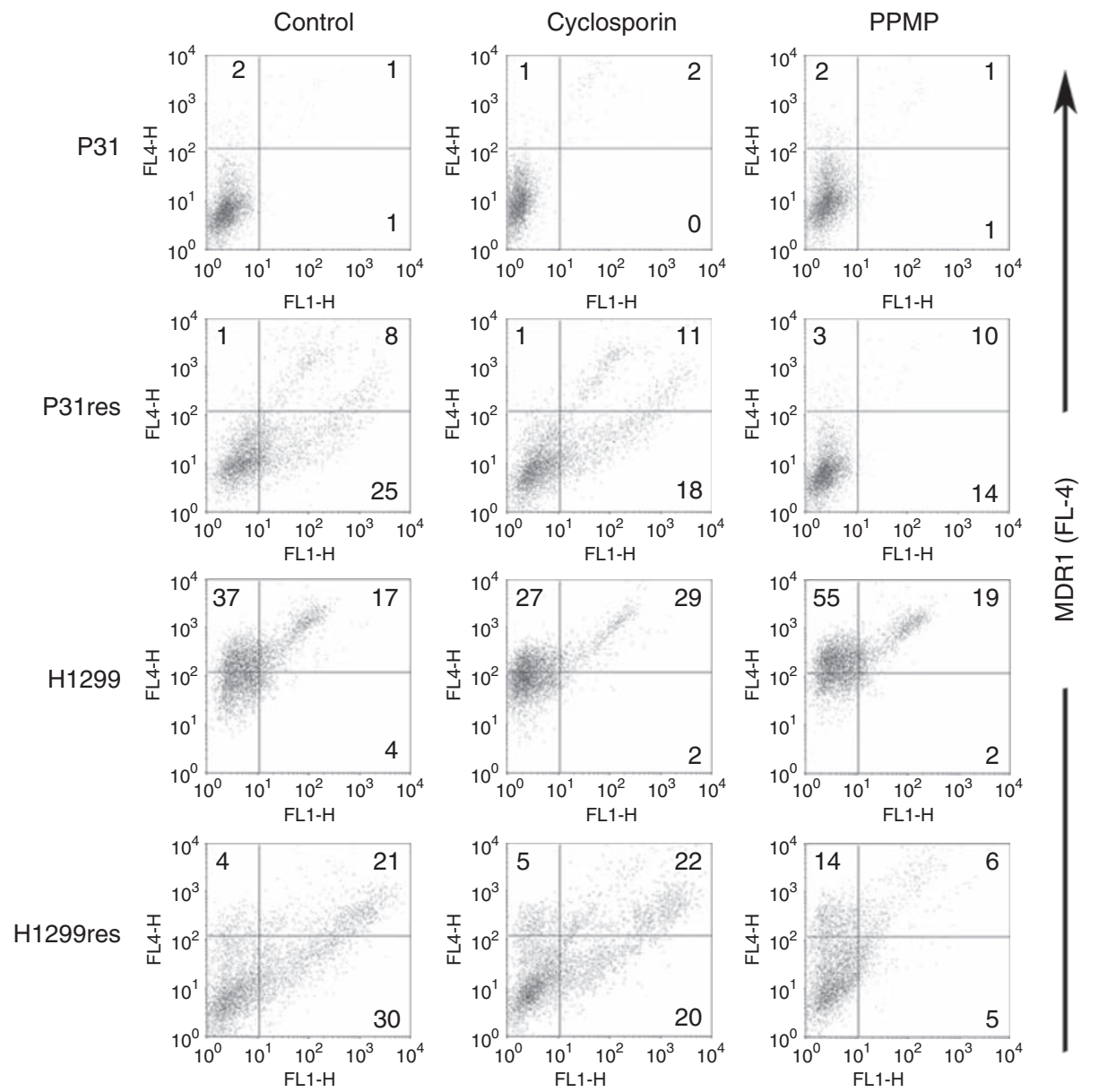

Figure 5 Flow cytometry analysis of Gb3- and MDRI/PgP expression of P3I and HI299 cells and their cisplatin-resistant sub-lines incubated with $10 \mu \mathrm{mol} \mathrm{I}{ }^{-1}$ cyclosporin A or $2 \mu \mathrm{mol} \mathrm{I}{ }^{-1}$ PPMP for $72 \mathrm{~h}$. Representative results are shown and the percentage mean $(n=3)$ of stained cells is noted in each dot plot.

noticeably inhibit MDR1/PgP expression in any of the cell types, but possibly the expression of $\mathrm{Gb} 3$ in the resistant sub-lines, whereas PPMP, as expected markedly, reduced not only Gb3 expression in the resistant cell sub-lines, but also of MDR1/PgP expression, especially of the cells of the resistant cell lines with also high expression of Gb3 (Figure 5).

\section{VT-1 and cisplatin induction of MPM cell DNA fragmentation}

The TUNEL-staining assay showed no increase of DNA fragmentation in P31 cells after exposure to $0.1 \mu \mathrm{gl}^{-1} \mathrm{VT}-1$ for $72 \mathrm{~h}$. A slight increase (to 17\%) in DNA fragmentation was, however, noted in 


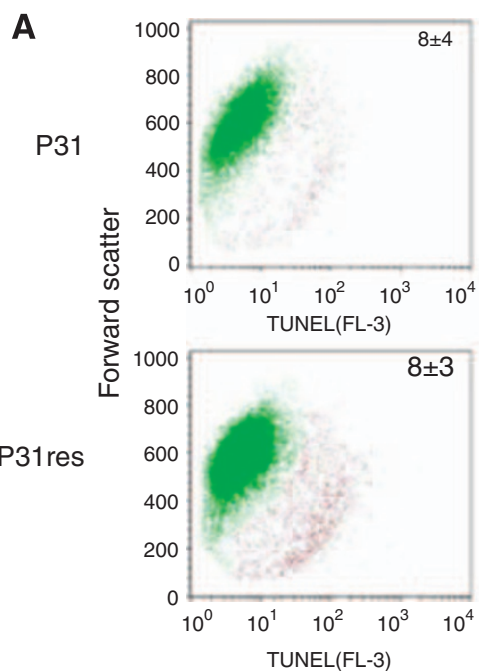

Cisplatin

Verotoxin-1
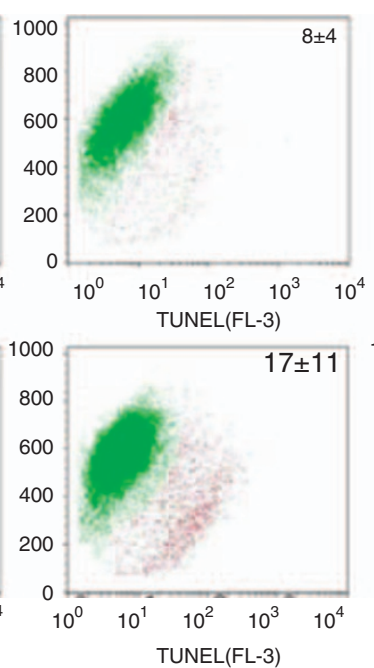

TUNEL staining
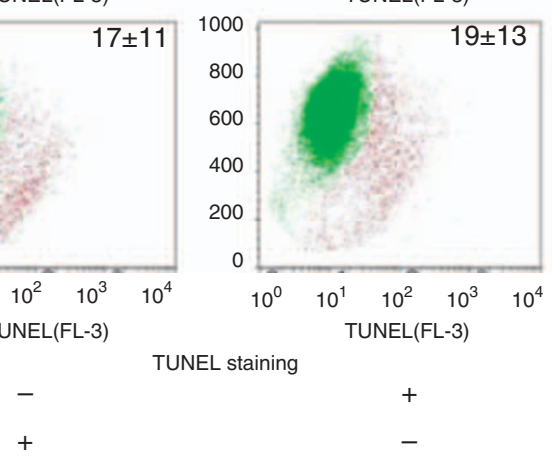
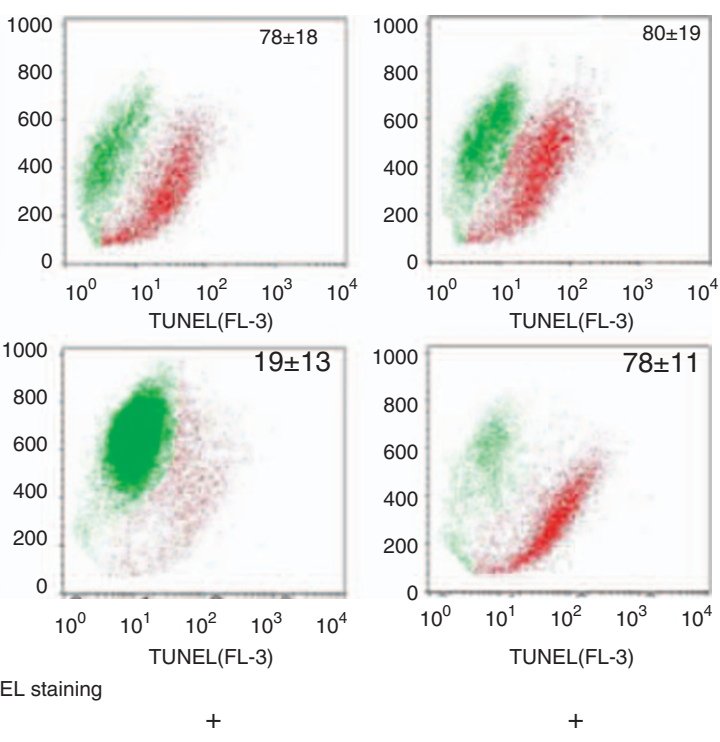

$+$
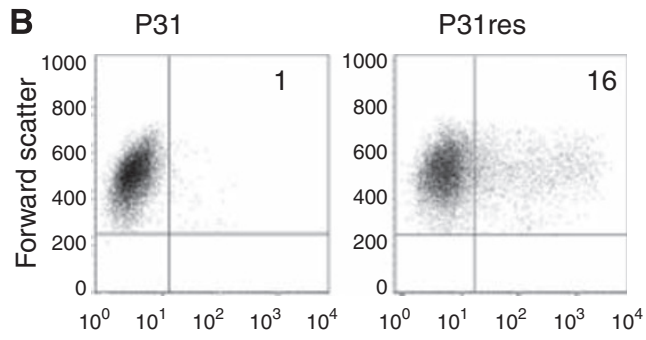

Gb3 (FL1)
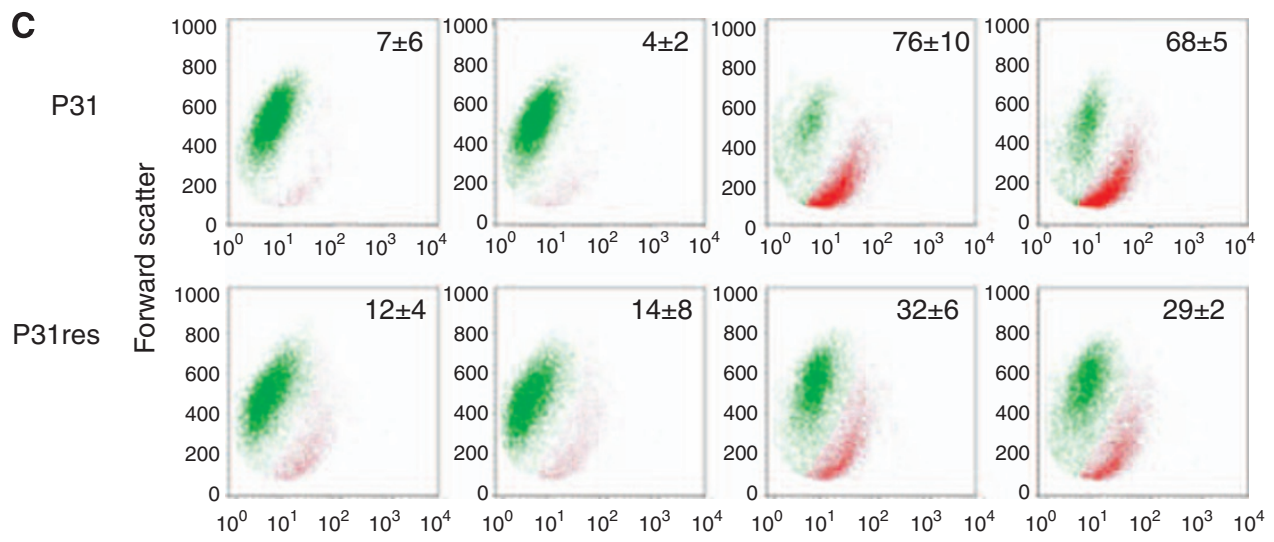

$\begin{array}{ll}\text { Cisplatin } & - \\ \text { Verotoxin-1 } & -\end{array}$
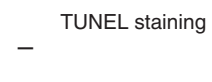

Figure 6 (A) Flow cytometry analysis of TUNEL staining in P3I cells after $72 \mathrm{~h}$ incubation with $5 \mathrm{mg} \mathrm{I}^{-1}$ cisplatin and $0.1 \mu \mathrm{g} \mathrm{I}^{-1} \mathrm{VT}$ - I, alone or in combination. Green dots indicate unstained cells and red TUNEL-stained cells. The percentage mean \pm s.d. $(n=3)$ of TUNEL-stained cells is noted in the dot plots. (B) Flow cytometry analysis of Gb3 expression in P3I and P3 I res cells after $72 \mathrm{~h}$ incubation with $2 \mu$ mol ${ }^{-1}$ PPMP. The percentage mean \pm s.d. $(n=3)$ of Gb3-stained cells is noted in each dot plot. $(\mathbf{C})$ Flow cytometry analysis of TUNEL staining in P3 I and P3 I res cells pre-incubated with $2 \mu$ mol I ${ }^{-}$ PPMP, and after $72 \mathrm{~h}$ incubation with $5 \mathrm{mgl}^{-1}$ cisplatin and $0.1 \mu \mathrm{gl}^{-1} \mathrm{VT}-\mathrm{I}$, alone or in combination. Green dots indicate unstained cells and red TUNELstained cells. The percentage mean \pm s.d. $(n=3)$ of TUNEL-stained cells is noted in each dot plot. Representative results are shown in $\mathbf{A}-\mathbf{C}$

the P31res cells (Figure 6A). Cisplatin $\left(5 \mathrm{mgl}^{-1}\right)$ was sufficient to induce massive (to 78\%) DNA fragmentation in P31 cells, whereas there was no or limited effect $(19 \%)$ in the resistant sub-line (P31res). The proportion of P31res cells with DNA fragmentation was dramatically increased (to $78 \%$ of the cells) by combined exposure to $5 \mathrm{mgl}^{-1}$ cisplatin and $0.1 \mu \mathrm{gl} 1^{-1} \mathrm{VT}-1$, but no further effect than that of cisplatin alone was noted in the P31 cells (Figure 6A). 
Inhibition of Gb3 expression eradicates VT-1 super-additive effect on cisplatin-induced TUNEL staining of cisplatin-resistant MPM cells

Exposure to $2 \mu \mathrm{moll}^{-1}$ PPMP for $72 \mathrm{~h}$ significantly reduced, but did not completely inhibit Gb3 expression in P31res cells (Figure 6B). PPMP treatment for $72 \mathrm{~h}$ eradicated DNA fragmentation induced by VT-1 $\left(0.1 \mu \mathrm{gl}^{-1}\right)$ in combination with cisplatin in P31 cells (Figure 6C). However, PPMP treatment seemed to sensitise P31res cells to the induction of apoptosis by $5 \mathrm{mgl}^{-1}$ cisplatin alone and possibly by itself induced low levels of DNA fragmentation (Figure 6C). Gb3 expression and TUNEL staining of P31 cells were unaffected by PPMP preincubation (Figure 6B and $\mathrm{C}$ ).

\section{VT-1 and cisplatin induction of MPM cell caspase activity}

When we studied signal transduction to apoptosis, the enzyme activity assays for caspase- $3,-8$, and -9 showed cisplatin-induced activation of caspase- 3 and -9 in P31cells, but not in P31res cells. VT-1 $\left(0.1 \mu \mathrm{g}^{-1}\right)$ activated caspase-3 in P31res, but had no effect on P31cells (Figure 7). When cisplatin was combined with VT-1, no further activation of caspase activity was noted except for slight increase of caspase-3 activity in P31res cells.

\section{Phosphorylation of MAPK proteins}

Western blotting was used to study expression of proteins potentially involved in apoptosis signalling of MPM cells after $24 \mathrm{~h}$ exposure to $5 \mathrm{mgl}^{-1}$ cisplatin with or without $0.1 \mu \mathrm{gl}^{-1} \mathrm{VT}-1$. An involvement of the stress-activated MAPK signalling pathway with increased phosphorylation of JNK and MKK3/6 was noted. JNK and MKK3/6 were phosphorylated after cisplatin exposure in P31cells, but not in P31res cells, whereas VT-1 induced phosphorylation in P31res cells, but not in P31cells. MKK3/6 phosphorylation was further augmented by the combination of cisplatin and VT-1 compared with VT-1 alone (Figure 8). Antibodies against AKT, p-Akt (ser473 and tyr308), Bad, p-Bad (ser 136 and 112), Bid, Bim, MCL-1, MKK-3, p-MKK3/6, P44/42, p-P44/42, or PUMA were all tested without conclusive protein expression changes after cisplatin and/or VT-1 exposure (data not shown).

\section{DISCUSSION}

This study shows the presence of the VT-1 cell receptor Gb3 in acquired-cisplatin resistance in both MPM and NSCLC cells. Furthermore, we show that cisplatin sensitises the cells to VT-1, leading to a potential treatment approach. Owing to the frequency of and the rapid acquirement of cisplatin resistance in the clinical setting, effective measures to circumvent this major treatment obstacle are of outmost importance.

We showed that cisplatin can up-regulate Gb3 expression in MPM and NSCLC cells and thus sensitise the cells to VT-1-induced cytotoxicity. The increased proportion of Gb3-expressing cells after cisplatin treatment suggests that cisplatin induces Gb3 expression in cancer cells, that cisplatin preferentially eradicates cell with low Gb3 expression, and that Gb3 expression is linked to acquired-cisplatin resistance. We could also correlate increased expression of Gb3 of cisplatin-resistant MPM and NSCLC cells to increased expression of MDR1/PgP. Gb3 and MDR1/PgP have recently been found to be partially co-localised in MDR1/PgPexpressing cells, and Gb3-containing lipid rafts are important for intracellular MDR1/PgP surface trafficking (De Rosa et al, 2008). The increased expression of MDR $1 / \mathrm{PgP}$ in cisplatin resistance could, therefore, parallel increased Gb3 expression, as MDR1/PgP acts as a glycolipid translocase involved in the biosynthesis of glycolipids such as Gb3 (De Rosa et al, 2008). However, we found
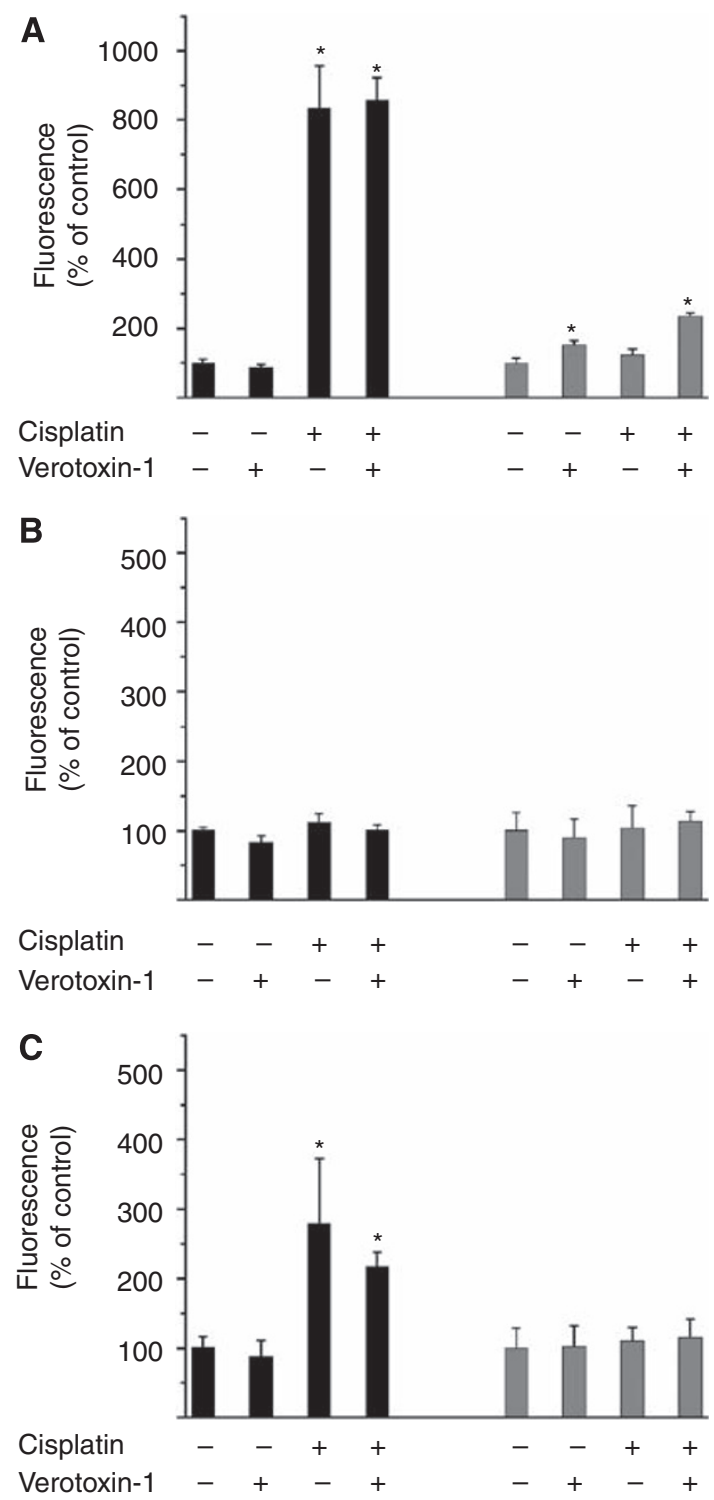

Figure 7 (A) Caspase-3, (B) -8, and (C) -9 enzyme activity in P3I (black bars) and P3/res cells (grey bars) after $24 \mathrm{~h}$ incubation with $5 \mathrm{mgl}^{-1}$ cisplatin and $0.1 \mu \mathrm{gI}^{-1} \mathrm{VT}$-I, alone or in combination. Significant enzyme activity differences $(P<0.05)$ compared with untreated control is indicated (*). Mean \pm s.d. $(n=3)$.

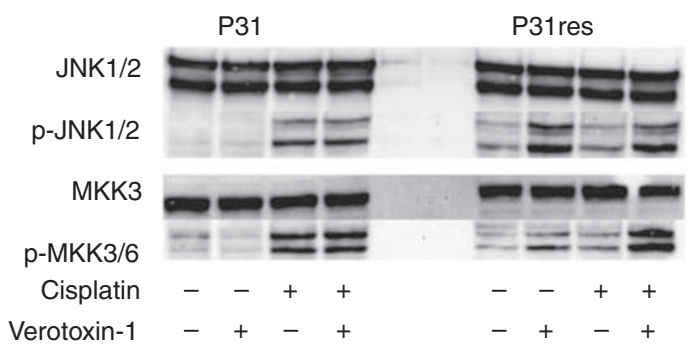

Figure 8 Western blot analysis of total and phosphorylated JNKI/2 and MKK3/6 protein expression of $\mathrm{P} 3 \mathrm{I}$ and $\mathrm{P} 3 \mathrm{I}$ res cells after $24 \mathrm{~h}$ incubation with $5 \mathrm{mgl}^{-1}$ cisplatin and $0.1 \mu \mathrm{g}^{-1} \mathrm{VT}-\mathrm{I}$, alone or in combination. Representative blot $(n=3)$.

no effect of the MDR1/PgP inhibitors verapamil or cyclosporin A on the expression of MDR1/PgP in either cell line, but PPMP reduced $\mathrm{Gb} 3$ expression in resistant sub-line cells and interestingly 
also particularly of the Gb3-expressing fraction that was induced when the mother cell line was made cisplatin resistant. Further studies on the interrelationship between multidrug-resistant cell expression of drug efflux pumps and Gb3 on cisplatin-resistant MPM and NSCLC cells are ongoing.

The reduced amount of Gb3-expressing cells after VT-1 treatment confirms that high-Gb3-expressing and cisplatin-resistant cells are sensitive to VT-1. Even though VT-1 alone had limited effect on the whole-cell population, it is of interest to note that there was a strong super-additive effect of combined cisplatin and VT-1 treatment in cisplatin-resistant MPM cells. The high sensitivity of the NSCLC cells to VT-1 despite a modest Gb3 expression implies that Gb3 expression, though necessary, does not mediate VT-1 cytotoxicity alone. The increased expression of Gb3 parallel to MDR1/PgP expression after induced cisplatin resistance with ensuing sensitivity to VT-1 cytotoxicity needs further investigation. It is in this context, however, that intriguing blockade of Gb3 synthesis eradicated VT-1-induced apoptosis as well as re-sensitised P31res cells to the induction of apoptosis by cisplatin alone.

We continued the study by investigating cisplatin- and VT-1induced cell death signal pathways in the MPM cells. TUNELlabelled DNA-fragmentation analysis showed an induced increase of apoptosis that correlated well with cell cytotoxicity and confirmed that the cisplatin-resistant sub-line was significantly less sensitive to cisplatin-induced apoptosis. Despite that neither VT-1 nor cisplatin induced more than limited amount of apoptosis in the cisplatin-resistant sub-line, the combined treatment induced similar levels of apoptosis as seen with cisplatin treatment alone of the more cisplatin-sensitive parental cell line. The lack of cisplatininduced DNA fragmentation in the resistant sub-line correlated with the results from the caspase activity assays, as there was no activation of either caspase-3, -8 , or -9 despite significant activation of caspases- 3 and -9 in the parental cell line. VT-1 did, however, slightly activate caspase- 3 activity in the resistant cells, which was further increased by combined VT-1/cisplatin treatment. This could explain the increased DNA fragmentation, especially as it has been shown that low levels of caspase- 3 activity is enough to induce apoptosis in these cells (Johansson et al, 2008).
PPMP inhibition of Gb3 synthesis confirmed that Gb3 is essential for VT-1-enhanced cisplatin-induced apoptosis.

Western blot was used to elucidate the signal transduction pathways to apoptosis of VT-1 and cisplatin alone and combined. By using antibodies specific for phosphorylated proteins of the MAPK pathway, we found that MKK3/6 and JNK was phosphorylated after cisplatin treatment in the cisplatin-sensitive cells, but not in the corresponding sub-lines with acquired-cisplatin resistance. VT-1 induced phosphorylation of MKK3/6, which was enhanced when VT-1 was combined with cisplatin. MKK3/6 is known to activate P38 (Derijard et al, 1995; Han et al, 1996), and P38 as well as JNK has been shown to promote apoptosis in response to cellular stress (Kim et al, 2006). Treatment of cells with chemical inhibitors or siRNA targeting p38 was recently shown to specifically inhibit VT-1 transport to the Golgi and reduced VT-1 toxicity (Walchli et al, 2008), and VT-1 prolonged JNK and p38 MAPK activation of macrophage-like cells (Lee et al, 2007). The MAPK pathway is thus involved in proapoptotic signalling of VT-1 in stressed cell systems and the pathway is also involved in cisplatin-induced apoptosis and induced cisplatin resistance (Brozovic and Osmak, 2007). Targeting the MAPK signalling pathway could, therefore, be an additional way to reduce cisplatininduced tumour cells resistance. We have earlier shown JNK phosphorylation in response to VT-1 treatment also in glioma cell lines (Johansson et al, 2006).

The treatment obstacle of acquired-cisplatin resistance in malignant plural mesothelioma and other cancers makes it necessary to find new strategies to overcome resistance. We have shown an increased expression of Gb3 in induced cisplatin-resistant MPM and NSCLC cells and a possible relation to multidrug resistance. Our results encourage the idea that Gb3-targeted therapy could be a possible approach and VT-1 either as holotoxin or by use of toxin sub-units could present a viable tool for this.

\section{ACKNOWLEDGEMENTS}

This study was supported by grants from the Swedish Cancer Society, the Cancer Research Foundation in Northern Sweden, and the Medical Faculty, Umeå University, Umeå Sweden.

\section{REFERENCES}

Andrews PA, Howell SB (1990) Cellular pharmacology of cisplatin: perspectives on mechanisms of acquired resistance. Cancer Cells 2: $35-43$

Arab S, Rutka J, Lingwood C (1999) Verotoxin induces apoptosis and the complete, rapid, long-term elimination of human astrocytoma xenografts in nude mice. Oncol Res 11: 33-39

Brognard J, Clark AS, Ni Y, Dennis PA (2001) Akt/protein kinase B is constitutively active in non-small cell lung cancer cells and promotes cellular survival and resistance to chemotherapy and radiation. Cancer Res 61: 3986-3997

Brozovic A, Osmak M (2007) Activation of mitogen-activated protein kinases by cisplatin and their role in cisplatin-resistance. Cancer Lett 251: $1-16$

Cvijic ME, Yang WL, Chin KV (1998) Cisplatin sensitivity in cAMPdependent protein kinase mutants of Saccharomyces cerevisiae. Anticancer Res 18: $3187-3192$

De Rosa MF, Ackerley C, Wang B, Ito S, Clarke DM, Lingwood C (2008) Inhibition of multidrug resistance by adamantylgb3, a globotriaosylceramide analog. J Biol Chem 283: $4501-4511$

Derijard B, Raingeaud J, Barrett T, Wu IH, Han J, Ulevitch RJ, Davis RJ (1995) Independent human MAP-kinase signal transduction pathways defined by MEK and MKK isoforms. Science 267: $682-685$

Endo Y, Tsurugi K, Yutsudo T, Takeda Y, Ogasawara T, Igarashi K (1988) Site of action of a Vero toxin (VT2) from Escherichia coli O157:H7 and of Shiga toxin on eukaryotic ribosomes. RNA N-glycosidase activity of the toxins. Eur I Biochem 171: 45-50
Gariepy J (2001) The use of Shiga-like toxin 1 in cancer therapy. Crit Rev Oncol Hematol 39: 99-106

Han J, Lee JD, Jiang Y, Li Z, Feng L, Ulevitch RJ (1996) Characterization of the structure and function of a novel MAP kinase kinase (MKK6). J Biol Chem 271: $2886-2891$

Ishitoya S, Kurazono H, Nishiyama H, Nakamura E, Kamoto T, Habuchi T, Terai A, Ogawa O, Yamamoto S (2004) Verotoxin induces rapid elimination of human renal tumor xenografts in SCID mice. J Urol 171: 1309-1313

Jacewicz M, Feldman HA, Donohue-Rolfe A, Balasubramanian KA, Keusch GT (1989) Pathogenesis of Shigella diarrhea. XIV. Analysis of Shiga toxin receptors on cloned HeLa cells. J Infect Dis 159: $881-889$

Jemal A, Murray T, Samuels A, Ghafoor A, Ward E, Thun MJ (2003) Cancer statistics, 2003. CA Cancer J Clin 53: 5-26

Johansson D, Johansson A, Behnam-Motlagh P (2008) alpha-Toxin of Staphylococcus aureus overcomes acquired cisplatin-resistance in malignant mesothelioma cells. Cancer Lett 265: 67-75

Johansson D, Johansson A, Grankvist K, Andersson U, Henriksson R, Bergstrom P, Brannstrom T, Behnam-Motlagh P (2006) Verotoxin-1 induction of apoptosis in Gb3-expressing human glioma cell lines. Cancer Biol Ther 5: $1211-1217$

Kasibhatla S, Tseng B (2003) Why target apoptosis in cancer treatment? Mol Cancer Ther 2: 573-580

Kim BJ, Ryu SW, Song BJ (2006) JNK- and p38 kinase-mediated phosphorylation of Bax leads to its activation and mitochondrial translocation and to apoptosis of human hepatoma HepG2 cells. J Biol Chem 281: $21256-21265$ 
Kovbasnjuk O, Mourtazina R, Baibakov B, Wang T, Elowsky C, Choti MA, Kane A, Donowitz M (2005) The glycosphingolipid globotriaosylceramide in the metastatic transformation of colon cancer. Proc Natl Acad Sci USA 102: 19087- 19092

Krishan A, Fitz CM, Andritsch I (1997) Drug retention, efflux, and resistance in tumor cells. Cytometry 29: 279-285

LaCasse EC, Bray MR, Patterson B, Lim WM, Perampalam S, Radvanyi LG, Keating A, Stewart AK, Buckstein R, Sandhu JS, Miller N, Banerjee D, Singh D, Belch AR, Pilarski LM, Gariepy J (1999) Shiga-like toxin-1 receptor on human breast cancer, lymphoma, and myeloma and absence from CD34(+) hematopoietic stem cells: implications for ex vivo tumor purging and autologous stem cell transplantation. Blood 94: $2901-2910$

Leard LE, Broaddus VC (2004) Mesothelial cell proliferation and apoptosis. Respirology 9: 292-299

Lee SY, Cherla RP and Tesh VL (2007) Simultaneous induction of apoptotic and survival signaling pathways in macrophage-like THP-1 cells by Shiga toxin 1. Infect Immun 75: 1291-1302

Lingwood CA, Khine AA, Arab S (1998) Globotriaosyl ceramide (Gb3) expression in human tumour cells: intracellular trafficking defines a new retrograde transport pathway from the cell surface to the nucleus, which correlates with sensitivity to verotoxin. Acta Biochim Pol 45: 351-359

Lingwood CA, Law H, Richardson S, Petric M, Brunton JL, De Grandis S, Karmali M (1987) Glycolipid binding of purified and recombinant Escherichia coli produced verotoxin in vitro. J Biol Chem 262: 8834-8839

Liu LZ, Zhou XD, Qian G, Shi X, Fang J, Jiang BH (2007) AKT1 amplification regulates cisplatin resistance in human lung cancer cells through the mammalian target of rapamycin/p70S6K1 pathway. Cancer Res 67: 6325-6332

Marklund SL, Westman NG, Lundgren E, Roos G (1982) Copper- and zinccontaining superoxide dismutase, manganese-containing superoxide dismutase, catalase, and glutathione peroxidase in normal and neoplastic human cell lines and normal human tissues. Cancer Res 42: 1955-1961

O'Brien AD, Tesh VL, Donohue-Rolfe A, Jackson MP, Olsnes S, Sandvig K, Lindberg AA, Keusch GT (1992) Shiga toxin: biochemistry, genetics, mode of action, and role in pathogenesis. Curr Top Microbiol Immunol 180: $65-94$

Ohmichi M, Hayakawa J, Tasaka K, Kurachi H, Murata Y (2005) Mechanisms of platinum drug resistance. Trends Pharmacol Sci 26: $113-116$

Olsnes S, Sandvig K (1988) How protein toxins enter and kill cells. Cancer Treat Res 37: 39-73

Parkin DM (2001) Global cancer statistics in the year 2000. Lancet Oncol 2: $533-543$

Rose PE, Clark AJ (1989) Haematology of the haemolytic uraemic syndrome. Blood Rev 3: 136-140

Salhia B, Rutka JT, Lingwood C, Nutikka A, Van Furth WR (2002) The treatment of malignant meningioma with verotoxin. Neoplasia 4: 304-311 Sandvig K, Grimmer S, Lauvrak SU, Torgersen ML, Skretting G, van Deurs B, Iversen TG (2002) Pathways followed by ricin and Shiga toxin into cells. Histochem Cell Biol 117: $131-141$

Saxena SK, O’Brien AD, Ackerman EJ (1989) Shiga toxin, Shiga-like toxin II variant, and ricin are all single-site RNA N-glycosidases of $28 \mathrm{~S}$ RNA when microinjected into Xenopus oocytes. J Biol Chem 264: 596-601

Siddik ZH (2003) Cisplatin: mode of cytotoxic action and molecular basis of resistance. Oncogene 22: $7265-7279$

Vingert B, Adotevi O, Patin D, Jung S, Shrikant P, Freyburger L, Eppolito C, Sapoznikov A, Amessou M, Quintin-Colonna F, Fridman WH, Johannes L, Tartour E (2006) The Shiga toxin B-subunit targets antigen in vivo to dendritic cells and elicits anti-tumor immunity. Eur J Immunol 36: $1124-1135$

Walchli S, Skanland SS, Gregers TF, Lauvrak SU, Torgersen ML, Ying M, Kuroda S, Maturana A, Sandvig K (2008) The mitogen-activated protein kinase p38 links Shiga toxin-dependent signaling and trafficking. Mol Biol Cell 19: 95 - 104

Zhou S-F (2008) Structure, function and regulation of P-glycoprotein and its clinical relevance in drug disposition. Xenobiot 38: $802-832$

Zwelling LA, Anderson T, Kohn KW (1979) DNA-protein and DNA interstrand cross-linking by cis- and trans-platinum(II) diamminedichloride in L1210 mouse leukemia cells and relation to cytotoxicity. Cancer Res 39: 365-369 